\title{
Complete pathological response to neoadjuvant pemetrexed/ cisplatin in combination with regional hyperthermia in a patient with sarcomatoid peritoneal mesothelioma Stephan Kruger ${ }^{\mathrm{a}}$, Martin K. Angele ${ }^{\mathrm{b}}$, Simone Reu ${ }^{\mathrm{d}}$, Karl Sotlar ${ }^{\mathrm{d}}$, Anno Graser ${ }^{\mathrm{c}}$, Michael Haas ${ }^{a}$, Markus Albertsmeier ${ }^{b}$, Hans-Joachim Stemmler ${ }^{a}$, Volker Heinemann ${ }^{\mathrm{a}}$, Lars H. Lindner ${ }^{\mathrm{a}, *}$ and Stefan Boeck ${ }^{\mathrm{a}, *}$
}

\begin{abstract}
Diffuse malignant peritoneal mesothelioma (DMPM) is a rare disease. Although most patients eligible for surgery undergo cytoreductive surgery in combination with hyperthermic intraperitoneal chemotherapy, the role of perioperative systemic chemotherapy still remains undefined. Here we report the case of a 52-year-old female patient with advanced sarcomatoid DMPM. After five cycles of systemic pemetrexed and cisplatin, along with two cycles of regional hyperthermia, tumor resection with histomorphological examination showed a complete pathological response. We therefore conclude that there is a subgroup of DMPM patients that might benefit from systemic neoadjuvant chemotherapy with pemetrexed and cisplatin. Anti-Cancer Drugs 25:854-856 (c) 2014 Wolters Kluwer Health | Lippincott Williams \& Wilkins.
\end{abstract}

\section{Introduction}

Diffuse malignant peritoneal mesothelioma (DMPM) is a rare disease accounting for $\sim 10 \%$ of all malignant mesotheliomas diagnosed in the USA [1]. The three major histological subtypes of DMPM are epithelial, sarcomatoid, and mixed subtypes. The epithelial subtype, the most common type of DMPM, has a considerably better prognosis than the sarcomatoid and mixed subtypes [2]. Other favorable prognostic factors are female sex, age below 50 years, a low score in the peritoneal cancer index measuring tumor burden, and the absence of lymph node or extra-abdominal metastases [3,4]. Owing to the low frequency of DMPM, no standard treatment approach exists. Most patients present with DMPM confined to the abdominal cavity with only marginal infiltration of surrounding organs. Hence, in many cases cytoreductive surgery, followed by hyperthermic intraperitoneal chemotherapy (HIPEC) is performed; systemic chemotherapy is mainly administered to patients ineligible for radical surgery or with dismal prognostic factors like nonepithelial subtype and high tumor burden [5]. In pleural mesothelioma, pemetrexed and cisplatin (PEM/CIS) represent an established treatment option; however, in DMPM systematic data on the efficacy of this regimen are still lacking [5]. Here we report the case of a 52-year-old female patient who was diagnosed with sarcomatoid DMPM and received preoperative treatment with PEM/ CIS in combination with regional hyperthermia.

\section{Anti-Cancer Drugs 2014, 25:854-856}

Keywords: cisplatin, hyperthermia, mesothelioma, pemetrexed

Departments of a Internal Medicine III and Comprehensive Cancer Center, Klinikum Grosshadern, ' ${ }^{\mathrm{G}}$ General, Visceral, Transplantation, Vascular and Thoracic Surgery, Klinikum Grosshadern, ${ }^{\circ}$ Clinical Radiology, Klinikum Grosshadern and ${ }^{\mathrm{d} D e p a r t m e n t}$ of Pathology, Ludwig-Maximilians-University of Munich, Munich, Germany

Correspondence to Stefan Boeck, MD, Department of Internal Medicine III and Comprehensive Cancer Center, Klinikum Grosshadern, Ludwig-MaximiliansUniversity of Munich, Marchioninistr. 15, D-81377 Munich, Germany Tel: + 49897095 2208; fax: + 49897095 5256;

e-mail: stefan.boeck@med.uni-muenchen.de

* Lars H. Lindner and Stefan Boeck contributed equally to the writing of this article.

Received 19 December 2013 Revised form accepted 17 February 2014

\section{Case report}

A 52-year-old white woman was referred to our cancer center in January 2013 for further work-up of an abdominal mass. Initial computed tomography (CT) imaging showed an $11.6 \times 7.6 \mathrm{~cm}$ intraperitoneal tumor mass with multiple smaller intraperitoneal lesions. Partly extending into the retroperitoneum, the tumor infiltrated the left kidney, colon, and small intestine (Fig. 1). Histomorphological investigation of a tumor sample obtained by CT-guided biopsy showed malignant neoplasm predominantly composed of spindle cells with a strong immunohistochemical expression of vimentin, a moderate expression of pan-cytokeratin, and a focal expression of mesothelin (with a lack of calretinin expression), compatible with the diagnosis of sarcomatoid peritoneal mesothelioma (Fig. 2a). Given the advanced stage of the disease, our multidisciplinary tumor board recommended preoperative chemotherapy with PEM $\left(500 \mathrm{mg} / \mathrm{m}^{2}\right)$ on day 1 plus CIS $\left(40 \mathrm{mg} / \mathrm{m}^{2}\right)$ in combination with regional hyperthermia on days 1 and 3 and a subsequent evaluation after four to six cycles of chemotherapy with regard to salvage surgery. A dosage reduction of both agents to $70 \%$ was necessary in cycle 3 because of pronounced leukocytopenia. Regional hyperthermia was poorly tolerated and had to be discontinued in cycle 3. Upon CT restaging after three cycles in April 2013, with no objective change in tumor size, two additional dose-reduced (70\%) cycles of PEM/CIS were administered. After five cycles of chemotherapy, the patient's general condition had significantly worsened with 
mucositis (grade II, according to National Cancer InstituteCommon Terminology Criteria for Adverse Events v. 3.0), pronounced leukocytopenia and thrombocytopenia (maximum grade IV), and progressive renal insufficiency (grade III). At this point, CT imaging without intravenous contrast showed only a minor decrease in tumor burden. As chemotherapy had been poorly tolerated and seemingly led only to minor objective tumor regression, our multidisciplinary tumor board recommended attempting surgical resection. Upon surgical exploration of the abdomen, the tumor was categorized as resectable by multivisceral

Fig. 1

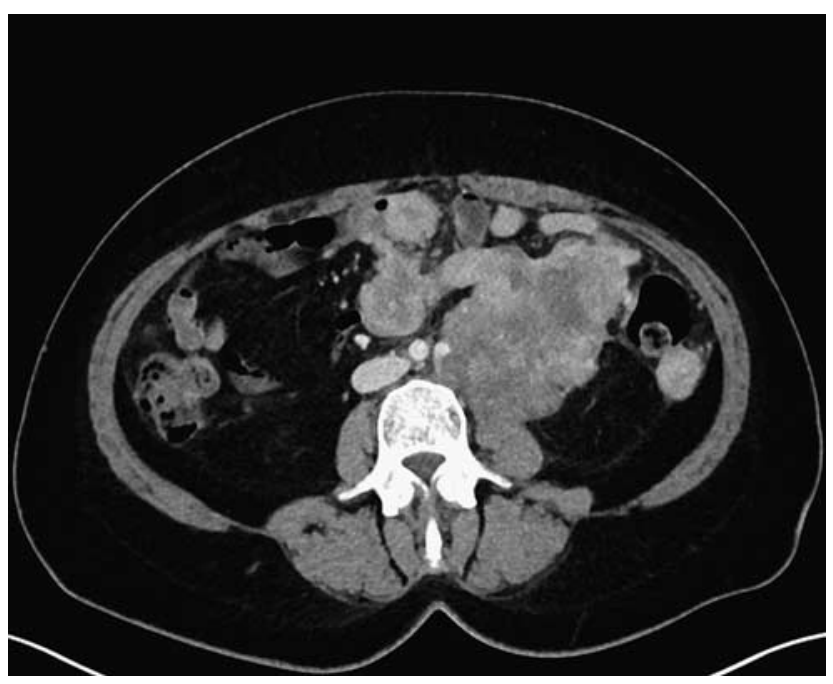

Computed tomography image of peritoneal mesothelioma at initial diagnosis. resection. Thus, en-bloc tumor extirpation, partial resection of the small intestine, left nephrectomy, and left hemicolectomy were performed in July 2013. No peritonectomy was performed. Upon histomorphological examination of the resected specimen, no vital tumor cells were found (Fig. 2b). Further, six lymph nodes analyzed were also tumor free. Therefore, response to preoperative treatment was classified as a complete pathological response. After surgery, our multidisciplinary tumor board thus recommended a standardized follow-up schedule without further adjuvant treatment. On the last examination in February 2014, the patient had recovered well from surgery and an ${ }^{18} \mathrm{~F}$-fluorodeoxyglucose/PET-CT scan without intravenous contrast (because of persistent renal insufficiency) showed no evidence of residual vital tumor tissue or tumor recurrence.

\section{Discussion}

In many cases DMPM is treated with cytoreductive surgery, followed by HIPEC, which have been hypothesized to prolong overall survival and disease free survival [6]. However, for patients initially ineligible for surgery only limited data on alternative treatment strategies exist. The use of different CIS-based systemic chemotherapy regimens as perioperative or palliative treatment for DMPM has been reported previously $[7,8]$. The PEM/CIS combination has been shown to effectively prolong overall survival in patients with pleural mesothelioma in a randomized phase III trial [9]. To date, however, no prospective study on the activity of PEM/ CIS in DMPM has been conducted. Hence information on the activity of PEM/CIS in DMPM can only be derived from its extended access program. The reported objective

Fig. 2
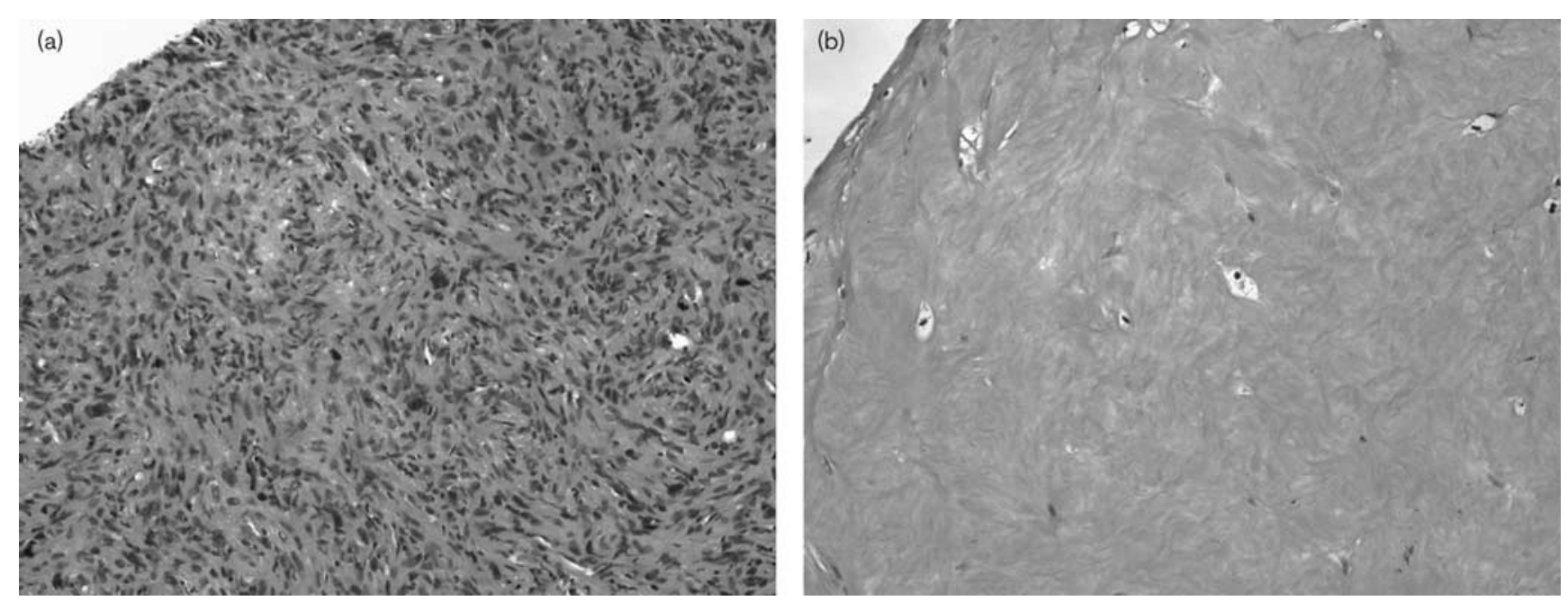

Hematoxylin and eosin staining of (a) a tumor biopsy at initial diagnosis showing sarcomatoid mesothelioma and (b) resected tumor tissue showing complete pathological response (magnification: $\times 200$ ). 
response rates (complete or partial remission) to treatment with PEM and PEM/CIS were 13 and 20-25\%, respectively $[8,10]$. On the basis of these findings, PEM/ CIS is used as palliative chemotherapy for DMPM. Yet, for patients undergoing cytoreductive surgery and HIPEC, a recent retrospective analysis by Deraco et al. [7] found no additional benefit with regard to overall survival for perioperative chemotherapy with PEM/CIS or other regimens. In contrast, our case suggests that there could be a subgroup of patients with tumors initially deemed as unresectable who might benefit from preoperative systemic chemotherapy and consecutive surgery. As conventional CT imaging did not adequately assess response to preoperative chemotherapy in the reported case, evaluation of metabolic response by ${ }^{18} \mathrm{~F}$-fluorodeoxyglucose/PET imaging could potentially be considered as a more appropriate staging tool for DMPM patients receiving chemotherapy. Besides evaluating alternative treatment strategies like regional hyperthermia in combination with systemic chemotherapy, future studies should also try to identify patients who might benefit most from perioperative chemotherapy to improve the unfavorable prognosis of patients suffering from DMPM.

\section{Acknowledgements Conflicts of interest}

There are no conflicts of interest.

\section{References}

1 Rodriguez D, Cheung MC, Housri N, Koniaris LG. Malignant abdominal mesothelioma: defining the role of surgery. J Surg Oncol 2009; 99:51-57.

2 Husain AN, Colby T, Ordonez N, Krausz T, Attanoos R, Beasley MB, et al. Guidelines for pathologic diagnosis of malignant mesothelioma: 2012 update of the consensus statement from the International Mesothelioma Interest Group. Arch Pathol Lab Med 2013; 137:647-667.

3 Mirabelli D, Roberti S, Gangemi M, Rosato R, Ricceri F, Merler E, et al. Survival of peritoneal malignant mesothelioma in Italy: a populationbased study. Int J Cancer 2009; 124:194-200.

4 Yan TD, Deraco M, Elias D, Glehen O, Levine EA, Moran BJ, et al. A novel tumor-node-metastasis (TNM) staging system of diffuse malignant peritoneal mesothelioma using outcome analysis of a multi-institutional database. Cancer 2011; 117:1855-1863.

5 Kindler HL. Peritoneal mesothelioma: the site of origin matters. In: Dizon DS editor. American Society Clinical Oncology educational book. 33rd ed. Alexandria: American Society of Clinical Oncology; 2013. pp. 182-188.

6 Yan TD, Deraco M, Baratti D, Kusamura S, Elias D, Glehen O, et al. Cytoreductive surgery and hyperthermic intraperitoneal chemotherapy for malignant peritoneal mesothelioma: multi-institutional experience. J Clin Oncol 2009; 27:6237-6242.

7 Deraco M, Baratti D, Hutanu I, Bertuli R, Kusamura S. The role of perioperative systemic chemotherapy in diffuse malignant peritoneal mesothelioma patients treated with cytoreductive surgery and hyperthermic intraperitoneal chemotherapy. Ann Surg Oncol 2013; 20:1093-1100.

8 Janne PA, Wozniak AJ, Belani CP, Keohan ML, Ross HJ, Polikoff JA, et al. Open-label study of pemetrexed alone or in combination with cisplatin for the treatment of patients with peritoneal mesothelioma: outcomes of an expanded access program. Clin Lung Cancer 2005; 7:40-46.

9 Vogelzang NJ, Rusthoven JJ, Symanowski J, Denham C, Kaukel E, Ruffie P et al. Phase III study of pemetrexed in combination with cisplatin versus cisplatin alone in patients with malignant pleural mesothelioma. J Clin Oncol 2003; 21:2636-2644.

10 Carteni G, Manegold C, Garcia GM, Siena S, Zielinski CC, Amadori D, et al. Malignant peritoneal mesothelioma-Results from the International Expanded Access Program using pemetrexed alone or in combination with a platinum agent. Lung Cancer 2009; 64:211-218. 